\title{
Synthesizing Reversible Circuits for Irreversible Functions
}

\author{
D. Michael Miller ${ }^{1}$ \\ Robert Wille ${ }^{2}$ \\ Gerhard W. Dueck ${ }^{3}$ \\ ${ }^{1}$ Department of Computer Science, University of Victoria, Canada \\ ${ }^{2}$ Group for Computer Architecture (Prof. Rolf Drechsler), University of Bremen, Germany \\ ${ }^{3}$ Faculty of Computer Science, University of New Brunswick, Canada \\ mmiller@uvic.ca, rwille@informatik.uni-bremen.de, gdueck@unb.ca
}

\begin{abstract}
Many reversible circuit synthesis procedures have been proposed. A common feature of most methods is that the initial specification must be a completely-specified reversible function. However, often the desired functionality is a, possibly incompletely-specified, irreversible function. In this paper, we consider how to fully automate the process of synthesizing a reversible function given an irreversible specification with particular emphasis on how to embed an irreversible function into a reversible specification. Systematic procedures are presented and results for benchmark problems show the methods produce very good results compared to earlier methods.
\end{abstract}

\section{INTRODUCTION}

The synthesis of reversible circuits has received much attention [1]-[17] due to the application of these techniques in low-power design and emerging (e.g. quantum, optical, DNA) computing technologies. These synthesis methods generally take a completely-specified reversible function as the starting point. Hence, to realize an irreversible function, which is most often the objective, usually requires that the function is embedded in a larger reversible specification. Such an embedding is not unique and the choice of embedding can have a very significant effect on the quality of the resulting circuit.

The process of embedding an irreversible function into a reversible specification requires the addition of constant inputs and garbage outputs as well as the assignment of don't-cares (DC) to ensure reversibility. For small functions, a good embedding can often be found manually based on knowledge of the structure of the irreversible function. In contrast, this becomes harder and often prohibitive as the function size grows.

In this paper, we consider the synthesis of a reversible circuit for an irreversible function to consist of three steps:

1) Embed the target irreversible function in a completelyspecified reversible function,

2) Synthesize a circuit for this reversible function, and

3) Perform post-synthesis optimization.

In particular, we consider the embedding issue and the circuit synthesis as separate steps so that any of the previously presented reversible synthesis methods can be applied. However, we also show how knowledge of the nature of the embedding can be used to simplify the synthesis process. Post-synthesis optimization is here restricted to the application of templates as described in [8].

The embedding problem requires the addition of extra lines and the assignment of DC conditions to achieve a completely-specified reversible function. The first is straightforward and we add the minimal number of lines possible [18]. Three methods for automatically assigning the DC are described and compared.

Other approaches to DC assignment when embedding an irreversible function in a reversible specification can be found in [5], [12], [17]. The method in [5] requires a very high number of garbage lines while the method in [12] generates circuits using gates with a high number of controls. The approach in [17] is of note in that it applies to both reversible and certain types of quantum gates. However, it is potentially computationally very expensive as it uses genetic algorithms and results are presented only for small circuits. A SAT-based approach providing exact solutions for small circuits can be found in [19].

Both the embedding and the synthesis approaches employ heuristics and it is not surprising that the results can be very sensitive to the ordering of the function outputs relative to the function inputs. For an $n$-output function there are $n$ ! output permutations and it is thus not feasible to synthesize a circuit for each of them. Therefore, we employ a method based on the 'sifting' method used to find good variable orderings in decision diagrams. This approach examines on the order of $n^{2}$ output permutations.

The main contributions of this paper are the study of embedding techniques and the effect of output permutation including the new sifting-based output permutation algorithm. Details of synthesizing a circuit from the reversible specification are not the main focus, and for clarity we consider algorithms employing multi-control Toffoli gates. However, the methods discussed are equally applicable when synthesis methods targeting other reversible gates are used.

After reviewing the necessary background in Section II, we show how irreversible functions can be embedded into reversible specifications in Section III. The DC assignment problem is addressed in Section IV. Then, the synthesis approaches employed in our work are briefly reviewed in Section V. The output permutation via sifting is discussed 
in Section VI. The paper ends with experimental results in Section VII and conclusions and suggestion for further research in the final section.

\section{BACKGROUND}

This section provides the necessary background for the development in this paper. Readers wishing more in-depth treatment should consult the references.

\section{Definition 1:}

1) An n-variable Boolean function $f(X), X=$ $\left\{x_{1}, x_{2}, \ldots, x_{n}\right\}$ is a mapping $f: B^{n} \rightarrow B$ with $B=\{0,1\}$.

2) An n-input, m-output Boolean function is a system of Boolean functions $y_{i}=f_{i}(X), 1 \leq i \leq m$, that will be more compactly written as $F(X)$. Such a function is referred to as a multiple-output function. For simplicity, we will term an $n$-input, $m$-output function an $n \times m$ function.

3) The multiple-output function $F(X)$ is completelyspecified if the value of every output is defined for every assignment to the inputs, otherwise it is incompletely-specified.

4) $F(X)$ is a reversible function iff $n=m$, the function is completely-specified and it maps each input assignment to a unique output assignment. A function that is not reversible is termed irreversible.

We denote the truth table for an $n \times m$ function $F(X)$ as $\mathbf{F}$. This table has $2^{n}$ rows $\mathbf{F}_{0}, \mathbf{F}_{1}, \ldots$, corresponding to the assignments to the variables of $X$ ordered in the conventional manner with $x_{1}$ as the most significant variable. $\mathbf{F}_{i}$ will denote the $i^{\text {th }}$ row of the table and $\mathbf{F}_{i, j}$ the $j^{\text {th }}$ entry in that row, i.e. the assignment to $f_{j}$. We refer to $\mathbf{F}_{0}$ as the first row in the table, and say that $\mathbf{F}_{i}$ occurs earlier than $\mathbf{F}_{j}$ if $i<j$.

Property 1: Since a $p \times p$ reversible function $F$ is a permutation mapping $2^{p}$ input patterns onto $2^{p}$ output patterns, $F^{-1}$ always exists and is the inverse permutation.

A Boolean function $f(X)$ can be expressed as a positive polarity Reed-Muller (PPRM) expansion $f(X)=$ $\bigoplus_{i=0}^{2^{n}-1} a_{i} P_{i}$ where $a_{i} \in\{0,1\}$ and $P_{i}$ is the product term containing those $x_{j}$ for which the $j^{\text {th }}$ position in the binary expansion of $i$ is 1 . Since we only deal with the PPRM case we will for brevity refer simply to RM.

The RM spectra of the size $\mathrm{n}$ identity function with outputs $y_{1}, y_{2}, \ldots y_{n}$ has a single nonzero coefficient $a_{2^{i-1}}$ for each $y_{i}$ with all other coefficients 0 .

Definition 2: The RM-distance of a reversible function from the identity function is the total number of coefficients for which its spectra differ from the spectra of the identity function for the same number of variables.

A reversible function can be realized by a circuit comprised of a cascade of reversible gates with no fan-out or feedback [20]. A reversible gate, itself realizes a reversible function. Many reversible gates have been proposed [20]. We
Table I

MCT Gate QuANTUM Cost.

\begin{tabular}{|c|c|c||c|c|c|}
\hline$m$ & $(n-m) \geq$ & cost & $m$ & $(n-m) \geq$ & cost \\
\hline 1 & & 1 & 8 & 5 & 62 \\
\hline 2 & & 1 & 8 & 1 & 100 \\
\hline 3 & & 5 & 8 & 0 & 253 \\
\hline 4 & & 13 & 9 & 6 & 74 \\
\hline 5 & 2 & 26 & 9 & 1 & 128 \\
\hline 5 & 0 & 29 & 9 & 0 & 509 \\
\hline 6 & 3 & 38 & 10 & 7 & 86 \\
\hline 6 & 1 & 52 & 10 & 1 & 152 \\
\hline 6 & 0 & 61 & 10 & 0 & 1021 \\
\hline 7 & 4 & 50 & $>10$ & $m-3$ & $12 m-34$ \\
\hline 7 & 1 & 80 & $>10$ & 1 & $24 m-88$ \\
\hline 7 & 0 & 125 & $>10$ & 0 & $2^{m}-3$ \\
\hline
\end{tabular}

here consider circuits composed of multiple control Toffoli gates which are defined as follows:

Definition 3: A multiple control Toffoli gate (MCT) with target line $x_{j}$ and control lines $\left\{x_{i_{1}}, x_{i_{2}}, . ., x_{i_{k}}\right\}$, maps $\left(x_{1}, x_{2}, . ., x_{j}, . ., x_{n}\right)$ to $\left(x_{1}, x_{2}, . ., x_{i_{1}} x_{i_{2}} . x_{i_{k}} \oplus x_{j}, . ., x_{n}\right)$. Note that all controls must be 1 for the target to be inverted.

An MCT gate with no controls always inverts the target line and is thus the well-known NOT gate. An MCT gate with a single control line is called a controlled-NOT gate. The case of two control lines is the original Toffoli gate.

We follow the normal convention of using a $\bigoplus$ to indicate the target line of an MCT gate and a to indicate the control connections. We will use the notation $M C T\{C ; t\}$ where $C$ is the set of controls and $t$ is the target.

The number of gates in a circuit is a simple but not very accurate measure of the circuit's complexity. Here, we will consider the quantum cost of a circuit which is taken as the sum of the quantum costs of the individual gates with no optimizations of neighboring gates. The quantum cost of an MCT gate is given in Table I (using the calculations introduced in [21] and further optimized in [18] and [22]). In this table, $m$ is the number of control and target lines for the gate and $n$ is the number of circuit lines.

A reversible circuit may have some number of constant inputs and garbage outputs defined as follows:

Definition 4: An constant input is additional to the primary function inputs and is set to a fixed value for the circuit to achieve the desired functionality.

Definition 5: A garbage output is additional to the primary function outputs and is thus a don't-care output.

Example 1: Fig. 1 shows a cascade realizing the full adder. This circuit consists of four MCT gates, one constant input, two garbage outputs, and has a quantum cost of 12 .

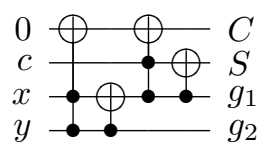

Figure 1. Toffoli gate full adder. 
Table II

EMbedding The Full AdDER INTO A REversible Function.

\begin{tabular}{ll} 
acxy & CSgg \\
\hline 0000 & $00--$ \\
0001 & $01--$ \\
0010 & $01--$ \\
0011 & $10--$ \\
0100 & $01--$ \\
0101 & $10--$ \\
0110 & $10--$ \\
0111 & $11--$ \\
1000 & --- \\
1001 & --- \\
1010 & --- \\
1011 & --- \\
1100 & --- \\
1101 & --- \\
1110 & --- \\
1111 & ---
\end{tabular}

(b)

\begin{tabular}{c|c} 
acxy & CSgg \\
\hline 0000 & 0000 \\
0001 & 0100 \\
0010 & 0101 \\
0011 & 1000 \\
0100 & 0110 \\
0101 & 1001 \\
0110 & 1010 \\
0111 & 1100 \\
1000 & 0001 \\
1001 & 0010 \\
1010 & 0011 \\
1011 & 0111 \\
1100 & 1011 \\
1101 & 1101 \\
1110 & 1110 \\
1111 & 1111
\end{tabular}

(c)

\section{EMBEDding OF IRREVERSIBLE FUnCTIONS}

In [18], it was shown that $g=\left\lceil\log _{2}(\mu)\right\rceil$ garbage outputs are required to embed a completely-specified irreversible function into a reversible function, where $\mu$ is the maximum number of times an output pattern is repeated in the truth table of the irreversible function. For an $n \times m$ irreversible function, this means the reversible function has $m+g$ outputs. Furthermore, $c$ constant inputs must be added such that $n+c=m+g$.

Once the garbage outputs and constant inputs are added, the critical issue is how to assign the DC in the expanded truth table as shown in the following example.

Example 2: Consider the adder function shown in Table II(a). This function has three inputs (the carry-in $\mathbf{c}$ as well as the two summands $\mathbf{x}$ and $\mathbf{y}$ ) and two outputs (the carryout $\mathbf{C}$ and the sum $\mathbf{S}$ ). This function is clearly irreversible since the number of inputs differs from the number of outputs. Since the output pattern 01 appears 3 times (as does the output pattern 10) adding one additional output to the function (leading to the same number of input and outputs) can not make the function reversible. In fact, $\left\lceil\log _{2}(3)\right\rceil=2$ garbage outputs must be added and hence a constant input must also be added. This is shown in Table II(b). Afterwards, the DC must be assigned so that the resulting function is reversible. One possible, albeit naive, embedding is shown in Table II(c). This embedding was found by assigning the garbage outputs to the patterns 00,01 , and 10 in order for each of the output patterns in the top half of the table and then completing the bottom half of the table using the remaining available output patterns in numerical order.

To appreciate the complexity of choosing a DC assignment, consider Table II(b). There are 4, 4, 3, 4, 2, 3, 2, and 4 choices for completing the DC in the top 8 rows of the table, respectively for a total of 9,216 choices. The bottom 8 rows of the table can then be completed in 8 ! ways. Lastly, the outputs can be permuted in $4 !=24$ ways. Combining these yields $9216 \times 40320 \times 24=8,918,138,880$ possible embeddings for this small example. Since the respective embedding may have a significant effect on the synthesis results (as will be shown in Section IV-D below), finding a 'good' assignment is an important non-trivial task.

\section{DC AssignMENT}

In this section, we present three novel methods for DC assignment to complete a reversible specification. We assume that if the irreversible function is incompletely-specified, then the DC in each truth table row will be preassigned to minimize the Hamming distance between the input and the output patterns. We always add the minimal number of garbage outputs and constant inputs to achieve a reversible specification. Lastly, all constant inputs are assigned the value 0 and are always added as the most significant inputs in the truth table. This leads to significant computational advantage as shown below and results in a circuit overhead of at most one NOT gate per constant input.

\section{A. A Greedy Algorithm}

The first two methods introduced here for assigning the DC are motivated by the basic operation of transformation based synthesis algorithms (see Section V-A). Those algorithms choose gates to map each row of the truth table to match the corresponding input pattern so that when all rows have been considered the table represents the identity function. It is thus reasonable to conjecture that assigning the DC to minimize the Hamming distance of the output patterns to the corresponding input patterns should help reduce the number of gates required. The first method proposed uses a simple greedy approach.

Algorithm 1: Greedy DC Assignment

Working from the top of the truth table downward:

1) For each distinct output assignment in the irreversible function, identify the target set of rows of the table containing that pattern. Then, determine the set of output assignments which are found by assigning the DC in all possible ways. The candidates are arranged in ascending numerical order.

2) For each row in the target set in turn, choose the first remaining candidate assignment with minimal Hamming distance to the input assignment for that row.

\section{B. Using the Hungarian Algorithm}

Let $S$ be the set of truth table rows sharing a common output pattern in the irreversible function and let $T$ be the set of possible assignments to the DC to complete those rows. $|T|=2^{g}$ where $g$ is the number of garbage lines added to permit the embedding of the irreversible function into a reversible one. The DC assignment problem is to associate each element of $S$ with a unique element from $T$. Let $K\left(S_{i}, T_{j}\right)$ be the 'cost' of associating the DC assignment $T_{j}$ with $S_{i} . K\left(S_{i}, T_{j}\right)$ is the Hamming distance between the completely-specified truth table output pattern and the corresponding input pattern when $S_{i}$ is completed using $T_{j}$. This formulation can be expressed in tabular form with a row for each $S_{i}$ and a column for each $T_{j}$ with each $K\left(S_{i}, T_{j}\right)$ in 
Table III

REVERSIBLE EMBEDDINGS FOR THE FULL ADDER.

\begin{tabular}{lc|c|c} 
acxy & $\begin{array}{c}\text { Greedy } \\
\text { CSgg }\end{array}$ & $\begin{array}{c}\text { XOR } \\
\text { CSgg }\end{array}$ & $\begin{array}{c}\text { Naive } \\
\text { CSgg }\end{array}$ \\
\hline 0000 & 0000 & 0000 & 0000 \\
0001 & 0101 & 0111 & 0100 \\
0010 & 0110 & 0110 & 0101 \\
0011 & 1011 & 1001 & 1000 \\
0100 & 0100 & 0100 & 0110 \\
0101 & 1001 & 1011 & 1001 \\
0110 & 1010 & 1010 & 1010 \\
0111 & 1111 & 1101 & 1100
\end{tabular}

\begin{tabular}{cc|c|c} 
acxy & $\begin{array}{c}\text { Greedy } \\
\text { CSgg }\end{array}$ & $\begin{array}{c}\text { XOR } \\
\text { CSgg }\end{array}$ & $\begin{array}{c}\text { Naive } \\
\text { CSgg }\end{array}$ \\
\hline 1000 & 1000 & 1000 & 0001 \\
1001 & 0001 & 1111 & 0010 \\
1010 & 0010 & 1110 & 0011 \\
1011 & 0011 & 0001 & 0111 \\
1100 & 1100 & 1100 & 1011 \\
1101 & 1101 & 0011 & 1101 \\
1110 & 1110 & 0010 & 1110 \\
1111 & 0111 & 0101 & 1111
\end{tabular}

the corresponding table entry. Assigning the DC to minimize the total Hamming distance is then a matter of choosing one entry in each row such that those entries appear in unique columns and such that the sum of the chosen entries is minimal. This is a standard assignment problem.

The Hungarian algorithm (also known as the KuhnMunkres and Munkres assignment algorithms) is a wellknown method [23] for solving the assignment problem in polynomial time. We have implemented this algorithms and results are reported below. The only issue of note here is that storing the potentially very large assignment matrix is avoided since Hamming distance is easily computed as needed - in fact more quickly than a matrix access.

\section{An XOR Based Method}

The third method we consider for DC assignment is based on the observation that for many functions a good embedding of an irreversible function into a reversible one is based upon setting the garbage outputs to XOR combinations of the primary inputs. This is often the case for arithmetic functions.

Algorithm 2: XOR-based DC Assignment

For $0 \leq i<2^{n}$ :

1) Set $k=i$.

2) Set $p=0, q=0$.

3) For $0 \leq j<n$ :

a) If $f_{j}$ is a garbage output set $q=q \oplus k_{j}$, and then set $p_{j}=q$.

b) Otherwise set $p_{j}=\boldsymbol{F}_{i j}$.

4) If $p$ represents an already assigned output pattern, increment $k$ and repeat from Step 2 .

5) $\operatorname{Set} \boldsymbol{F}_{i}=p$.

\section{Example}

Table III shows embeddings for the full adder using the greedy and the XOR based methods as well as the naive assignment from Table II(c). In this case, the assignment produced by the Hungarian Algorithm method is the same as the assignment for the Greedy method. The circuits for each assignment found using the transformation-based synthesis method [8] (see Section V-A) are shown in Fig. 2. These circuits clearly show the importance of a good DC assignment. The XOR-based method yields a circuit with quantum costs of only 13 while the others yield costs of 27 and 44.

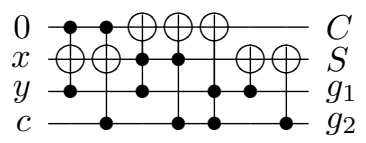

(a) Greedy assignment:

7 gates, quantum cost $=27 \quad 5$ gates, quantum cost $=13$

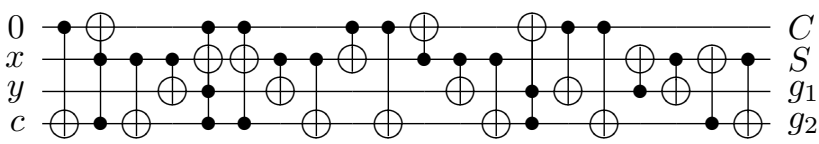

(c) Naive assignment: 20 gates, quantum cost $=44$

Figure 2. Circuits for the Embeddings in Table III.

\section{SYNTHESIS AFTER DC ASSIGNMENT}

After applying one of the above methods, or an alternative, for DC assignment to embed an irreversible function into a completely-specified reversible function, any reversible circuit synthesis method can be applied. We briefly outline two approaches used in the experimental evaluation later in this paper. Furthermore, a combined approach as well as a simplified synthesis method are proposed.

\section{A. Transformation Based Synthesis}

Transformation based synthesis for reversible functions was introduced in [7]. The basic idea is to traverse the truth table row by row beginning at the top (the $0^{\text {th }}$ ) row. At each step gates are selected to transform the output assignment to match the input assignment. These gates are chosen so that they don't alter rows earlier in the table. After all rows are processed the table represents the identity function and the sequence of gates identified in reverse order is a circuit implementing the given reversible function. The order of gates is reversed because the transformation of output patterns to match input patterns is identifying gates from the output side of the circuit.

Two improvements are possible based on the fact a completely-specified function always has an inverse. First, gates can be chosen to transform an input pattern to match an output pattern if that is cheaper than the other way round. Details can be found in [7]. The second improvement is based on the following result:

Theorem 1: [24] If the cascade of MCT gates $G_{1}, G_{2}, \ldots G_{k}$ realizes a reversible function $F$, then the reverse cascade $G_{k}, \ldots, G_{2}, G_{1}$ realizes the inverse function $F^{-1}$.

Thus, the transformation based approach can be applied to the inverse of the given function. Since heuristics are used, there can be a significant difference in circuit cost for a function and its inverse. This transformation method was adapted to use the Reed-Muller (RM) spectra of a totallyspecified reversible function in [8], [9]. 


\section{B. Reed-Muller Based Search Algorithm}

A second approach introduced in [8] is a very simple search method. At each step, by exhaustive enumeration, it selects the Toffoli gate whose application to the function specification results in the largest decrease of the RMdistance to the identity function (see Definition 2). If no gate application decreases the RM-distance to the identity, a gate is chosen that results in the minimal increase in the RM-distance. In both cases, if there is a tie between two or more gates, the gate with the smallest control set is chosen. If there is a tie based on the number of controls, the method selects the first gate in lexicographic order. The work in [8], [9] showed using RM spectra to be more effective than the Walsh spectrum, used in [25].

This simple search approach has two major drawbacks. First, the algorithm is not guaranteed to converge. Second, the number of possible MCT gates at each stage of the search can be prohibitive.

\section{A Combined Algorithm}

In [8], the non convergence issue was addressed by first applying the transformation based algorithm and to use the circuit produced as a bound when the search procedure is applied. A more effective alternative is outlined in the following algorithm:

Algorithm 3: Combined Synthesis Algorithm

1) Set size $=1$.

2) Consider all MCT gates with size -1 controls applied to the function specification, i.e. at the output side of the circuit.

3) Choose a gate which most reduces the RM-distance to the identity function. If there is a tie the first one encountered is used. Apply the gate. If the function has been mapped to the identity, stop; otherwise go back to Step 1.

4) If no gate can be found that decreases the RM-distance, increment size. If size $>\max$ Size go to Step 5, otherwise go to Step 2.

5) Apply the RM spectra-based transformation synthesis algorithm to complete the circuit.

This algorithm incorporates the advantages of the two basic approaches. Using searching first often identifies gates with few controls to begin the circuit that have a strong influence across the function specification. The control value size tends to limit the amount of searching required. We have found that setting sizeLimit $=5$ yields good results. Resorting to the transformation based approach when searching fails guarantees that a solution is found. The algorithm can be extended by applying bi-directional techniques in both the search and transformation based phases and can be applied to the function and its inverse.

\section{Simplified Synthesis}

As noted above, we require that all constant inputs take the value 0 and are always the most significant inputs in the truth table. This means that the rows of the irreversible function truth table are always embedded in the first rows of the reversible function truth table while the remaining rows are total DC. In particular, if the reversible function has $n$ primary inputs and $c$ constant inputs we care about the output for the first $2^{n}$ rows of the truth table. The remaining $\left(2^{c}-1\right) 2^{n}$ rows can be ignored.

Clearly, given the above construction, a transformation based synthesis method that works row by row from the top of the truth table can stop after transforming $2^{n}$ rows. Because of this, it is not necessary to complete a DC assignment beyond the $\left(2^{n}\right)^{t h}$ row.

It is not so obvious that this simplification also applies to both the RM transformation and RM search methods.

1) RM Transformation Synthesis: RM transformation synthesis can stop after the first $2^{n}$ rows as described above since, as was shown in [9], if the first $p$ rows of the spectra match the spectra of the identity, then the first $p$ rows in the truth table also match the identity.

2) RM Search Synthesis: Since we only care about the first $2^{n}$ rows, we can restrict the gate application to those rows. Furthermore, the RM-distance metric need only be computed over those rows. What goes on below them simply does not matter and has no affect on the first $2^{n}$ rows.

Note that when these simplifications are employed, bidirectional synthesis methods can not be applied because we are in fact not working with a completely-specified function and thus have no definition of its inverse. Our experiments show that stopping the synthesis after $2^{n}$ rows and avoiding circuitry to align DC rows outweighs the disadvantage of not being able to apply bidirectional techniques.

\section{OPTIMIZATIONS}

\section{A. Template Matching}

The application of templates to simplify a reversible circuit was introduced in [7] based on the work on circuit transformation rules in [26]. The approach has since been significantly refined [8], [27]. Basically, template matching searches a circuit for a set of gates that either occur together or can be moved to occur together, and then replaces those gates with a less costly set of gates with the same functionality. We here use the templates reported in [8] and use quantum cost as the metric.

Without going into detail, it should be clear that template application can be very computationally expensive due to the amount of searching required including finding appropriate gate repositionings. For that reason, we use a basic set of 14 templates. If the best possible circuit is sought, Dueck and Maslov's [28] 220 templates can be used.

\section{B. Output Permutation}

Assuming the correspondence between the inputs and outputs is not fixed a less costly circuit can often be found by reordering the outputs in the specification and resynthesizing. It is not practical to try all $p$ ! possibilities for a problem with $p$ outputs. We instead employ sifting as suggested in [29]. A significant new idea here is that 
rather than using sifting to position the outputs one by one in the order specified, we use the extent to which an output contributes to the RM-distance as a metric to order the sifting of the outputs. The following outlines the basic output sifting approach omitting specific detail on optimization and the avoidance of resynthesizing the same specification.

Algorithm 4: Output Sifting

For a specification with $p$ outputs (including garbage outputs):

1) Assign the DC using one of the methods above. Only the first $2^{n}$ rows need be assigned where $n$ is the number of non-constant inputs.

2) Repeat the following $p$ times:

a) Choose an as yet unsifted output $y_{k}$ that most contributes to the RM-distance between the specification and the identity function.

b) Reorder the specification $p$ times placing $y_{k}$ in each of the $p$ possible positions leaving the relative ordering of the other outputs unchanged. Resynthesize the circuit for each of these repositionings and keep track of the position of $y_{k}$ that gives the lowest quantum cost circuit.

c) Put $y_{k}$ into its best position and record the corresponding circuit as the best seen to date.

3) Report the best circuit found throughout the sifting process.

This algorithm considers $p^{2}$ of the $p$ ! output orderings so $p^{2}$ synthesis attempts are required. Hence the method is expensive to apply for functions with a large number of outputs but it is feasible for $p$ up to 14. Results presented below show it is quite effective.

\section{EXPERIMENTAL RESULTS}

Table IV presents results for the incompletelyspecified benchmark functions available in RevLib [30] (www.revlib.org). The number at the end of each benchmark name is a unique identifier of the pla-files from RevLib. Results are given for two synthesis methods (combined and transformation based) and for each of the DC assignment methods: greedy, Hungarian algorithm based, and XOR based. The MCT gate count and quantum cost are shown for each circuit. Furthermore, for each benchmark, the best results in terms of quantum cost are highlighted in bold. An AMD Athlon 3500+ with 1 GB of memory was used for our experiments. Every circuit in Table IV was found in less than $0.25 \mathrm{CPU}$ seconds.

The results show no DC assignment method and synthesis method pair is consistently the best. However, since the execution times are relatively short, it is feasible to run all combinations and choose the best circuit obtained. As can be clearly seen, this leads to significant reductions. For example, the synthesis results for function rd73_69 ranges from quantum cost of 1112 to quantum cost of 184 depending on the DC assignment method applied. An improvement of nearly one order of magnitude is achieved.

Table $\mathrm{V}$ shows the effect of the sifting based output permutation method described in Section VI-B followed by template matching from [8]. The benchmark functions from Table IV as well as a number of completely-specified reversible benchmark functions from RevLib are considered. The results labeled 'Initial Synthesis' are for the combined synthesis approach with XOR-based DC assignment used when needed. The section 'Output Permutation (sifting)' shows the results for the same synthesis approach when sifting is employed. The percentage improvement in the circuit quantum cost is shown with a blank entry indicating no improvement.

The section labeled 'Template Matching' shows the result of applying template matching (with 14 templates) to the best circuit found by sifting. Again the improvement shown is with respect to the circuit quantum cost.

The CPU seconds required for the initial synthesis, for output permutation optimization, and for template matching are shown with blank entries indicating the CPU time was less than 0.01 CPU seconds. The time is quite reasonable even for the larger circuits but the results indicate that the time will grow significantly as the number of circuit lines increases. This is largely due to the fact that our current implementation uses truth tables. As anticipated, the CPU requirement for synthesis is roughly $n \times n$ times the time required for the initial synthesis. Template matching is reasonably efficient but the time is significant for the circuits with a large number of gates. However, recall that we here use a set of 14 templates. The time is much higher if a large set of templates is used.

Table V also compares the circuits after template matching with prior results. Those marked with an asterisk are from [8], the others are the best MCT result from the RevLib web site as of March 2009. The quantum cost of our result is shown as a percentage of the cost of the prior result for each function. These comparisons show that the combination of methods described here produce very good results. Indeed there are some cases, where larger circuits are obtained, but recall, that we are comparing the results of our synthesis approach with the best ones obtained by various methods. Furthermore, the circuits quoted from RevLib sometimes uses more lines than the minimal which is what our methods use. If we add these lines and apply some transformations, our results come very close to the RevLib results and in some instances are better.

\section{CONCLUSIONS AND FUtURE RESEARCH}

The first contribution of this paper is the presentation of systematic and complete procedures to determine a reversible circuit for an irreversible function. The second contribution is showing that by assigning the constant inputs to 0 and placing them in the high order variable positions in the truth table, both the DC assignment and the synthesis tasks can be substantially simplified. The third contribution is a sifting method for finding a good output permutation which use the RM-distance metric to determine the order in which the outputs are sifted.

The presented results show that the methods discussed can be quite effective. However, as is often the case in complex 
Table IV

Comparison of DC Assignment Methods for Two Synthesis Algorithms

\begin{tabular}{|c|c|c|c|c|c|c|c|c|c|c|c|c|c|c|c|}
\hline \multirow{2}{*}{\multicolumn{4}{|c|}{ Benchmark }} & \multicolumn{6}{|c|}{ Combined Synthesis Algorithm } & \multicolumn{6}{|c|}{ Transformation Synthesis Algorithm } \\
\hline & & & & \multicolumn{2}{|c|}{ Greedy } & \multicolumn{2}{|c|}{ Hungarian } & \multicolumn{2}{|c|}{ XOR-based } & \multicolumn{2}{|c|}{ Greedy } & \multicolumn{2}{|c|}{ Hungarian } & \multicolumn{2}{|c|}{ XOR-based } \\
\hline & $\mathbf{n}$ & c & $\mathbf{g}$ & gates & cost & gates & cost & gates & cost & gates & cost & gates & cost & gates & cost \\
\hline decod24_10 & 4 & 2 & 0 & 7 & 11 & 7 & 11 & 7 & 11 & 7 & 11 & 7 & 11 & 7 & 11 \\
\hline rd32_19 & 4 & 1 & 2 & 5 & 13 & 5 & 13 & 5 & 13 & 5 & 17 & 5 & 17 & 5 & 17 \\
\hline 4gt10_22 & 5 & 1 & 4 & 3 & 47 & 3 & 47 & 4 & 40 & 3 & 47 & 3 & 47 & 3 & 47 \\
\hline 4gt11_23 & 5 & 1 & 4 & 1 & 5 & 1 & 5 & 4 & 12 & 1 & 5 & 1 & 5 & 1 & 5 \\
\hline $4 \mathrm{gt} 12 \_24$ & 5 & 1 & 4 & 3 & 55 & 3 & 55 & 6 & 62 & 3 & 55 & 3 & 55 & 3 & 55 \\
\hline 4gt13_25 & 5 & 1 & 4 & 1 & 13 & 1 & 13 & 3 & 27 & 1 & 13 & 1 & 13 & 1 & 13 \\
\hline 4gt4_20 & 5 & 1 & 4 & 6 & 58 & 6 & 58 & 9 & 65 & 7 & 79 & 7 & 79 & 7 & 79 \\
\hline 4gt5_21 & 5 & 1 & 4 & 3 & 19 & 3 & 19 & 6 & 30 & 3 & 19 & 3 & 19 & 3 & 19 \\
\hline $4 \bmod 5 \_8$ & 5 & 1 & 4 & 7 & 19 & 7 & 19 & 5 & 9 & 9 & 25 & 9 & 25 & 9 & 25 \\
\hline 4mod7_26 & 5 & 1 & 2 & 21 & 65 & 21 & 65 & 21 & 65 & 15 & 55 & 15 & 55 & 15 & 55 \\
\hline alu_9 & 5 & 0 & 4 & 9 & 65 & 16 & 72 & 28 & 224 & 12 & 64 & 16 & 68 & 32 & 252 \\
\hline mini-alu_84 & 5 & 1 & 3 & 22 & 110 & 26 & 114 & 25 & 125 & 22 & 98 & 28 & 108 & 22 & 110 \\
\hline one-two-three_27 & 5 & 2 & 2 & 9 & 33 & 9 & 33 & 9 & 33 & 9 & 33 & 9 & 33 & 9 & 33 \\
\hline decod24-enable_32 & 6 & 3 & 2 & 15 & 39 & 11 & 35 & 14 & 42 & 15 & 39 & 13 & 37 & 15 & 39 \\
\hline rd53_68 & 7 & 2 & 4 & 27 & 228 & 27 & 228 & 22 & 137 & 22 & 187 & 22 & 187 & 22 & 187 \\
\hline sym6̄_63 & 7 & 1 & 6 & 36 & 485 & 36 & 485 & 17 & 133 & 36 & 777 & 36 & 777 & 36 & 777 \\
\hline rd73_69 & 9 & 2 & 6 & 80 & 1112 & 80 & 1112 & 40 & 184 & 100 & 2187 & 100 & 2187 & 100 & 2187 \\
\hline sym9_71 & 10 & 1 & 9 & 76 & 1047 & 76 & 1047 & 51 & 573 & 210 & 4368 & 210 & 4368 & 210 & 4368 \\
\hline rd84_70 & 11 & 3 & 7 & 104 & 1823 & 104 & 1823 & 47 & 446 & 111 & 2100 & 111 & 2100 & 111 & 2100 \\
\hline
\end{tabular}

design problems and when heuristics are employed, no single method, or combination of methods, is universally the best. In practice, it is necessary to try different approaches. This can be costly since, while the DC assignment and synthesis methods are themselves relatively fast, the application of output permutation by sifting and template matching can be very costly consuming many minutes of CPU time. We are studying the benchmarks presented here and others to see if there exist properties that will predict which approach would be best for particular types of functions.

Our current implementations are limited as they store the truth tables and the RM spectra as integer vectors. We are in the process of migrating the methods to decision diagram implementations to improve both the efficiency and the size of problem that can be considered. Also, our implementation of sifting for output permutation is very simplistic.

We are investigating how to handle DC in the target irreversible function. One approach [5] is to use a minimizer to assign the DC but that tends to increase the output multiplicity and the number of garbage outputs and constant inputs that must be added. Our current approach is to assign the irreversible function DC to minimize the Hamming distance between the input and output patterns in each truth table row. Future research will consider other alternatives.

\section{REFERENCES}

[1] V. V. Shende, A. K. Prasad, I. L. Markov, and J. P. Hayes, "Reversible logic circuit synthesis," in Proc. Int'l Conf. on CAD, 2002, pp. 125132 .

[2] A. Agrawal and N. Jha, "Reversible logic synthesis," in Proc. Design, Automation and Test in Europe, 2004, pp. 1384-1385.

[3] J. Donald and N. K. Jha, "Reversible logic synthesis with Fredkin and Peres gates," J. Emergin Technology Computing Systems, vol. 4, no. 1 , pp. 1-19, 2008

[4] P. Gupta, A. Agrawal, and N. K. Jha, "An algorithm for synthesis of reversible logic circuits," IEEE Trans. on CAD, vol. 25, no. 11, pp. 2317-2330, Nov. 2006.
[5] Z. Guan, X. Qin, Z. Ge, and Y. Zhang, "Reversible synthesis with minimum logic function," in Proc. Conf. on Computational Intelligence and Security, vol. 2, 2006, pp. 968-971.

[6] P. Kerntopf, "A new heuristic algorithm for reversible logic synthesis," in Proc. Design Automation Conf., 2004, pp. 834-837.

[7] D. M. Miller, D. Maslov, and G. W. Dueck, "A transformation based algorithm for reversible logic synthesis," in Proc. Design Automation Conf., 2003, pp. 318-323.

[8] D. Maslov, G. W. Dueck, and D. M. Miller, "Techniques for the synthesis of reversible Toffoli networks," ACM Trans. Des. Autom. Electron. Syst., vol. 12, no. 4, pp. 42.1-42.28, 2007.

[9] D. Maslov and D. M. Miller, "Reed-Muller spectra based synthesis of reversible circuits using a quantum cost metric," in 7th International Symposium on Representations and Methodology of Future Computing Technologies (RM), 2005.

[10] M. Saeedi, M. Sedighi, and M. S. Zamani, "A novel synthesis algorithm for reversible circuits," in Proc. Int'l Conf. on CAD, 2007, pp. 65-68.

[11] M. Saeedi, M. S. Zamani, and M. Sedighi, "On the behavior of substitution-based reversible circuit synthesis algorithms: Investigation and improvement," in Proc. IEEE Computer Society Symp. on VLSI, 2007, pp. 428-436.

[12] K. Fazel, M. Thornton, and J. E. Rice, "ESOP-based Toffoli gate cascade generation," in Proceedings IEEE Pacific Rim Conference on Communications, Computers and Signal Processing (PACRIM), 2007, pp. 206-209.

[13] Z. Sasanian, M. Saeedi, M. Sedighi, and M. Zamani, "A cyclebased synthesis algorithm for reversible logic," in Proc. ASP Design Automation Conf., 2009, pp. 745-750.

[14] Y. Zheng and C. Huang, "A novel Toffoli network synthesis algorithm for reversible logic," in Proc. ASP Design Automation Conf., 2009, pp. 739-744.

[15] M. Saeedi, M. S. Zamani, and M. Sedighi, "Algebraic characterization of CNOT-baed quantum circuits with its application to logic synthesis," in Proc. European Conf. on Digital Systems Design, 2007, pp. 339-346.

[16] D. Yuan, B. Yan, L. WenTao, and G. Yi, "Research and implementation of reversible logic synthesis algorithm in digital system," in Proc. Computer-Aided Indust. Design and Conceptual Design, 2006, pp. 1-7.

[17] M. Mohammadi and M. Eshghi, "Heuristic methods to use don'tcares in automated design of reversible and quantum logic circuits," Quantum Information Processing, vol. 7, pp. 175-192, 2008. 
Table V

Effect of Output Permutation by Sifting Followed by Template Matching

\begin{tabular}{|c|c|c|c|c|c|c|c|c|c|c|c|c|c|c|c|}
\hline \multicolumn{2}{|l|}{ Benchmark } & \multicolumn{3}{|c|}{ Initial Synthesis } & \multicolumn{4}{|c|}{ Output Permutation (sifting) } & \multicolumn{4}{|c|}{ Template Matching } & \multicolumn{3}{|c|}{ Prior Results } \\
\hline & $\mathbf{n}$ & gates & cost & CPU & gates & cost & $\%$ & CPU & gates & cost & $\%$ & CPU & gates & cost & $\%$ \\
\hline 3_17_6 & 3 & 6 & 14 & & 6 & 14 & & & 6 & 14 & & & 6 & $14^{*}$ & $100.0 \%$ \\
\hline ex-1_82 & 3 & 4 & 8 & & 4 & 8 & & & 4 & 8 & & & 4 & 9 & $88.9 \%$ \\
\hline fredkin_3 & 3 & 3 & 7 & & 3 & 7 & & & 3 & 7 & & & 3 & 15 & $46.7 \%$ \\
\hline ham3 $\overline{28}$ & 3 & 5 & 9 & & 3 & 7 & $22.2 \%$ & & 3 & 7 & & & 5 & 9* & $77.8 \%$ \\
\hline miller_5 & 3 & 5 & 9 & & 5 & 9 & & & 5 & 9 & & & 5 & 17 & $52.9 \%$ \\
\hline peres_4 & 3 & 2 & 6 & & 2 & 6 & & & 2 & 6 & & & 2 & 6 & $100.0 \%$ \\
\hline toffoli_1 & 3 & 1 & 5 & & 1 & 5 & & & 1 & 5 & & & 1 & 5 & $100.0 \%$ \\
\hline 4_49_7 & 4 & 22 & 54 & & 16 & 36 & $33.3 \%$ & 0.01 & 16 & 36 & & 0.03 & 12 & 16 & $225.0 \%$ \\
\hline aj-e11_81 & 4 & 14 & 34 & & 13 & 33 & $2.9 \%$ & & 13 & 33 & & 0.02 & 10 & 30 & $110.0 \%$ \\
\hline decod24-enable_32 & 4 & 14 & 42 & & 10 & 34 & $19.0 \%$ & & 9 & 33 & $2.9 \%$ & 0.01 & 9 & 9 & $366.7 \%$ \\
\hline hwb4_12 & 4 & 17 & 29 & & 12 & 28 & $3.4 \%$ & 0.01 & 11 & 23 & $17.9 \%$ & 0.02 & 11 & $23^{*}$ & $100.0 \%$ \\
\hline rd32_19 & 4 & 5 & 13 & & 5 & 13 & & & 6 & 10 & $23.1 \%$ & & 4 & 12 & $83.3 \%$ \\
\hline toffoli_double_2 & 4 & 2 & 10 & & 4 & 8 & $20.0 \%$ & & 4 & 8 & & & 2 & 10 & $80.0 \%$ \\
\hline 4gt10_22 & 5 & 4 & 40 & & 4 & 40 & & & 5 & 37 & $7.5 \%$ & & 5 & 37 & $100.0 \%$ \\
\hline 4 gt11 23 & 5 & 4 & 12 & & 1 & 5 & $58.3 \%$ & & 1 & 5 & & & 4 & 8 & $62.5 \%$ \\
\hline 4gt12_24 & 5 & 6 & 62 & & 4 & 36 & $41.9 \%$ & & 4 & 36 & & & 5 & 41 & $87.8 \%$ \\
\hline 4gt13_25 & 5 & 3 & 27 & & 1 & 13 & $51.9 \%$ & & 1 & 13 & & & 3 & 15 & $86.7 \%$ \\
\hline 4gt4_20 & 5 & 9 & 65 & & 14 & 46 & $29.2 \%$ & & 13 & 45 & $2.2 \%$ & 0.03 & 6 & 54 & $83.3 \%$ \\
\hline $4 \mathrm{gt5} 221$ & 5 & 6 & 30 & & 4 & 16 & $46.7 \%$ & & 4 & 16 & & & 5 & 21 & $76.2 \%$ \\
\hline $4 \bmod 5 \_8$ & 5 & 5 & 9 & & 5 & 9 & & & 5 & 9 & & & 5 & $13^{*}$ & $69.2 \%$ \\
\hline $4 \bmod 7 \_26$ & 5 & 21 & 65 & & 18 & 62 & $4.6 \%$ & & 17 & 57 & $8.1 \%$ & 0.06 & 6 & 38 & $150.0 \%$ \\
\hline alu_9 & 5 & 28 & 224 & & 22 & 110 & $50.9 \%$ & & 23 & 107 & $2.7 \%$ & 0.08 & 6 & 14 & $764.3 \%$ \\
\hline hwb5_13 & 5 & 39 & 179 & & 48 & 136 & $24.0 \%$ & 0.07 & 46 & 126 & $7.4 \%$ & 0.26 & 24 & $114^{*}$ & $110.5 \%$ \\
\hline mini-alu_84 & 5 & 25 & 125 & & 21 & 97 & $22.4 \%$ & & 21 & 93 & $4.1 \%$ & 0.07 & 6 & 62 & $150.0 \%$ \\
\hline $\bmod 5 \mathrm{~d} 1 \_16$ & 5 & 7 & 15 & & 7 & 15 & & 0.02 & 7 & 11 & $26.7 \%$ & 0.01 & 7 & 11 & $100.0 \%$ \\
\hline $\bmod 5 \mathrm{~d} 2 \_17$ & 5 & 8 & 16 & & 9 & 13 & $18.8 \%$ & 0.02 & 9 & 13 & & 0.01 & 8 & 16 & $81.3 \%$ \\
\hline mod5mils_18 & 5 & 5 & 13 & & 5 & 13 & & 0.02 & 6 & 10 & $23.1 \%$ & & 5 & 13 & $76.9 \%$ \\
\hline one-two-three_27 & 5 & 9 & 33 & & 7 & 27 & $18.2 \%$ & & 7 & 27 & & 0.01 & 8 & 40 & $67.5 \%$ \\
\hline decod24_10 & 6 & 7 & 11 & & 6 & 10 & $9.1 \%$ & & 6 & 10 & & 0.01 & 9 & 21 & $47.6 \%$ \\
\hline graycode6_11 & 6 & 5 & 5 & & 5 & 5 & & 0.05 & 5 & 5 & & & 5 & 5 & $100.0 \%$ \\
\hline hwb6 14 & 6 & 169 & 1129 & 0.01 & 101 & 657 & $41.8 \%$ & 0.40 & 104 & 556 & $15.4 \%$ & 0.88 & 42 & $150 *$ & $370.7 \%$ \\
\hline mod5adder_66 & 6 & 21 & 121 & 0.01 & 21 & 121 & & 0.32 & 18 & 110 & $9.1 \%$ & 0.06 & 17 & 77 & $142.9 \%$ \\
\hline ham7_29 & 7 & 26 & 90 & 0.02 & 24 & 72 & $20.0 \%$ & 1.06 & 27 & 55 & $23.6 \%$ & 0.11 & 25 & $49^{*}$ & $112.2 \%$ \\
\hline hwb7_15 & 7 & 319 & 3189 & 0.03 & 299 & 2731 & $14.4 \%$ & 1.59 & 299 & 2450 & $10.3 \%$ & 3.40 & 331 & $2609^{*}$ & $93.9 \%$ \\
\hline mod10_86 & 7 & 33 & 110 & & 27 & 88 & $20.0 \%$ & 0.03 & 27 & 84 & $4.5 \%$ & 0.12 & 7 & 43 & $195.3 \%$ \\
\hline rd53_68 & 7 & 22 & 137 & & 23 & 64 & $53.3 \%$ & 0.03 & 22 & 59 & $7.8 \%$ & 0.10 & 16 & $67^{*}$ & $88.1 \%$ \\
\hline sym6_63 & 7 & 17 & 133 & & 16 & 107 & $19.5 \%$ & 0.04 & 18 & 93 & $13.1 \%$ & 0.06 & 15 & 62 & $150.0 \%$ \\
\hline hwb8_64 & 8 & 705 & 8489 & 0.10 & 375 & 5121 & $39.7 \%$ & 6.41 & 385 & 4516 & $11.8 \%$ & 5.46 & 748 & $6197^{*}$ & $72.9 \%$ \\
\hline urf2_73 & 8 & 894 & 10117 & 0.19 & 906 & 9283 & $8.2 \%$ & 11.73 & 900 & 8843 & $4.7 \%$ & 18.11 & 620 & 16152 & $54.7 \%$ \\
\hline hwb9-65 & 9 & 2023 & 23740 & 0.34 & 1709 & 21996 & $7.3 \%$ & 24.76 & 1677 & 20933 & $4.8 \%$ & 57.94 & 1959 & $20378^{*}$ & $102.7 \%$ \\
\hline rd73_69 & 9 & 40 & 184 & 0.01 & 31 & 112 & $39.1 \%$ & 0.56 & 29 & 90 & $19.6 \%$ & 0.15 & 20 & 76 & $118.4 \%$ \\
\hline urf1_72 & 9 & 2019 & 24674 & 0.77 & 1992 & 23471 & $4.9 \%$ & 62.44 & 1934 & 22703 & $3.3 \%$ & 76.97 & 1487 & 45855 & $49.5 \%$ \\
\hline urf5_76 & 9 & 929 & 19811 & 0.39 & 893 & 16082 & $18.8 \%$ & 37.12 & 913 & 14297 & $11.1 \%$ & 20.24 & 499 & 24253 & $58.9 \%$ \\
\hline sym9_71 & 10 & 51 & 573 & 0.03 & 38 & 324 & $43.5 \%$ & 2.95 & 39 & 229 & $29.3 \%$ & 0.24 & 21 & 94 & $243.6 \%$ \\
\hline urf3_75 & 10 & 3999 & 59719 & 2.38 & 4053 & 59325 & $0.7 \%$ & 255.51 & 3937 & 57365 & $3.3 \%$ & 340.37 & 2674 & 121716 & $47.1 \%$ \\
\hline rd84_70 & 11 & 47 & 446 & 0.05 & 42 & 441 & $1.1 \%$ & 5.86 & 50 & 280 & $36.5 \%$ & 0.47 & 15 & 112 & $250.0 \%$ \\
\hline cycle10_2_61 & 12 & 44 & 3423 & 4.86 & 34 & 1570 & $54.1 \%$ & 563.93 & 29 & 1212 & $22.8 \%$ & 0.18 & 19 & $1206^{*}$ & $100.5 \%$ \\
\hline plus63mod4096_79 & 12 & 26 & 4875 & 3.26 & 26 & 4875 & & 441.04 & 26 & 4875 & & 0.12 & 429 & 32539 & $15.0 \%$ \\
\hline plus127mod8192_78 & 13 & 27 & 9133 & 10.40 & 27 & 9133 & & 1673.83 & 27 & 9133 & & 0.12 & 910 & 73357 & $12.5 \%$ \\
\hline plus63mod8192_80 & 13 & 30 & 9185 & 10.42 & 30 & 9185 & & 1664.73 & 30 & 9185 & & 0.13 & 492 & 45025 & $20.4 \%$ \\
\hline
\end{tabular}

[18] D. Maslov and G. W. Dueck, "Reversible cascades with minimal garbage." IEEE Trans. on CAD, vol. 23, no. 11, pp. 1497-1509, 2004.

[19] R. Wille and D. Große, "Fast exact Toffoli network synthesis of reversible logic," in Proc. Int'l Conf. on CAD, 2007, pp. 60-64.

[20] M. Nielsen and I. Chuang, Quantum Computation and Quantum Information. Cambridge Univ. Press, 2000.

[21] A. Barenco, C. Bennett, R. Cleve, D. DiVinchenzo, N. Margolus, P. Shor, T. Sleator, J. Smolin, and H. Weinfurter, "Elementary gates for quantum computation," Physical Review A, vol. 52, pp. 34573467,1995

[22] D. Maslov, C. Young, D. M. Miller, and G. W. Dueck, "Quantum circuit simplification using templates," in Proc. Design, Automation and Test in Europe, 2005, pp. 1208-1213.

[23] F. S. Hillier and G. J. Lieberman, Introduction to Operations Research. McGraw-Hill Professional, 2005.

[24] D. Maslov, "Reversible logic synthesis," Ph.D. dissertation, University of New Brunswick, 2003.

[25] D. M. Miller and G. W. Dueck, "Spectral techniques for reversible logic synthesis," in 6th Int. Symp. on Representations and Methodology of Future Computing Technologies, 2003, pp. 56-62.

[26] K. Iwama, Y. Kambayashi, and S. Yamashita, "Transformation rules for designing CNOT-based quantum circuits," in Proc. Design Automation Conf., 2002, pp. 419-424.

[27] D. Maslov, G. W. Dueck, and D. M. Miller, "Fredkin/Toffoli templates for reversible logic synthesis," in Proc. Int'l Conf. on CAD, 2003, pp. 256-261.

[28] G. W. Dueck and D. Maslov, "Generation of multiple-control Toffoli network templates," in Proc. Int'l Workshop on Logic Synthesis, 2007, pp. 39-44.

[29] R. Wille, D. Große, G. W. Dueck, and R. Drechsler, "Reversible logic synthesis with output permutation," in Proc. Int'l Conf. on VLSI Design, 2009, pp. 189-194.

[30] R. Wille, D. Große, L. Teuber, G. W. Dueck, and R. Drechsler, "RevLib: An online resource for reversible functions and reversible circuits," in Int'l Symp. on Multi-Valued Logic, 2008, pp. 220-225, RevLib is available at www.revlib.org. 